\title{
The Social Determinants of Health
}

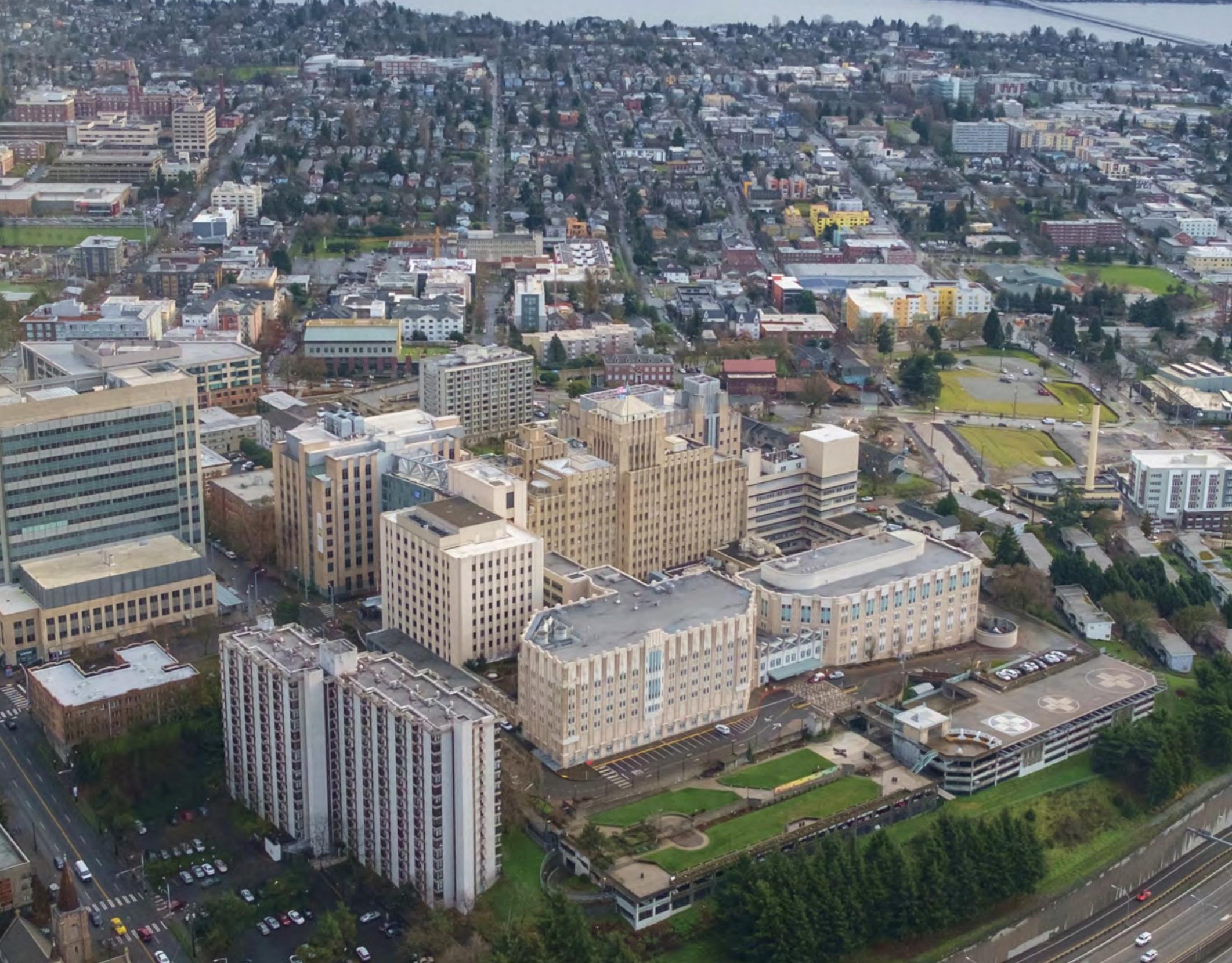




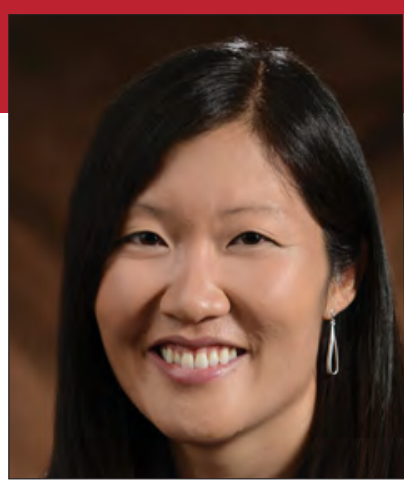

Antonia F. Chen, MD, MBA ${ }^{1}$ Moderator

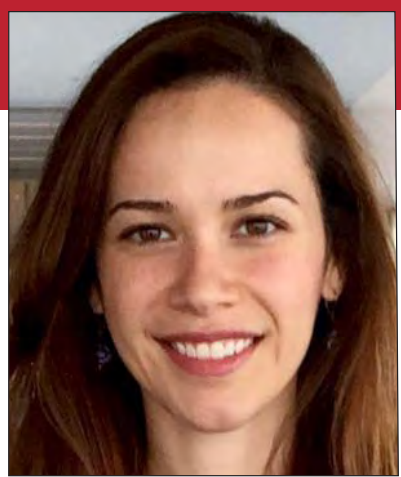

Norma A. Padrón, $\mathrm{MA}, \mathrm{MPH}, \mathrm{PhD}^{2}$

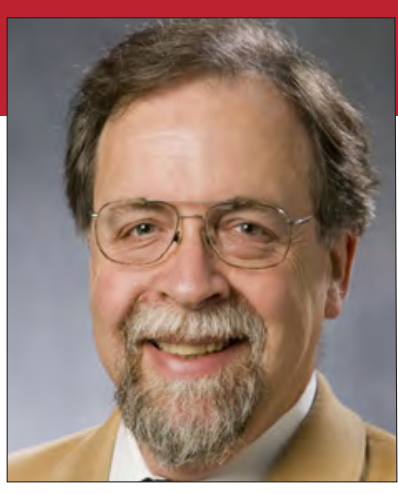

Charles M. Becker, PhD ${ }^{3}$

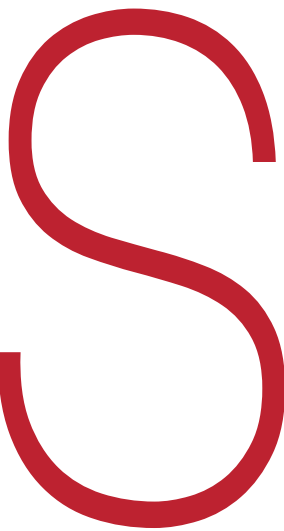

hould your zip code determine how long you live? It's been said zip codes predict life-span more accurately than genetic codes. When you look at the maps, the data are unavoidable ... and unacceptable.

Sadly, Philadelphia records the worst disparity-20 years. Baltimore is right behind. A child born today in one neighborhood will see the dawn of the 22 nd century. A child born a mile away is unlikely to make 2090-among the worst life expectancies in the world.

Our goal has been to reduce longevity disparities to within 5 years. Can we do that?

Here are the dots we have to connect, well enumerated by the participants in this roundtable on social determinants of health.

On the one hand, we understand social factors-obesity and nutrition, smoking, transportation, jobs, housing. At the same time, we have issues of access to "personal healthcare," what's called "amenable mortality."

We have to do better with both. It's time for healthcare delivery to step out-to shift the focus of diagnosis and treatment to the home.

'Rothman Institute and Sidney Kimmel Medical College, Thomas Jefferson University, Philadelphia, PA

${ }^{2}$ Associate Director, MLHS Center for Population Health Research at LIMR, Assistant Professor, College of Population Health, Thomas Jefferson University, Philadelphia, PA.

${ }^{3}$ Associate Chair and Research Professor, Department of Economics, Duke University, Durham, NC.

(c) Antonia F. Chen et al. 2017; Published by Mary Ann Liebert, Inc. This article is available under the Creative Commons License CC-BY-NC (http://creativecommons.org/licenses/by-nc/4.0). This license permits non-commercial use, distribution, and reproduction in any medium, provided the original work is properly cited. Permission only needs to be obtained for commercial use and can be done via RightsLink. 
One day, everyone-in any neighborhood - should be able to answer the question "Where do you get your healthcare?" with the answer "Where I am."

\section{- The future is hyper-local}

\section{Dr. Antonia F. Chen: What does your ideal U.S. healthcare delivery system in 2030 look like?}

Dr. Norma A. Padrón: Well, 2030 is actually not that far away, so I am going to keep my answer contingent on today's environment. I believe that alternative payment structures and alternative payment models will continue to develop, and I do believe that it is feasible that both commercial payers and providers can forge ahead, despite what currently seems like a shaky financial landscape for healthcare.

I also think that local health departments and health systems, as well as at the state level, will start taking stronger steps in public health. These public health and healthcare delivery partnerships, along with redesigned and aligned financial incentives in the form of alternative payment models, will have the potential to move the needle in advancing overall health and well-being.

\section{I believe that alternative payment structures and alternative payment models will continue to develop 99}

My take is that the U.S. healthcare delivery system in 2030 will be hyper-local. We'll see a lot more public-private partnerships between local departments of health and health systems and financial models that align incentives across sectors.

Dr. Charles M. Becker: The U.S. population is aging, but there is now a documented decline in population health improvements, especially in older middle-aged and young elderly individuals. I would be surprised if costs did not continue to rise as the population ages and as demand to reduce mortality emerges.

That will put tremendous pressure on the system. Because of this, it is going to be very difficult not to move toward a baseline healthcare minimum in a single-payer system, such as a Medicare for all.

However, the problem is that people who are younger and relatively healthy will not want to pay into a full cost-recovery single-payer system, and cost savings from these plans would be modest while demands will be high. My hunch is that the gap between multiple healthcare systems delineated by socioeconomic class is going to get so big that it is going to be untenable. 


\section{Healthcare for all? Here's how}

\section{Dr. Chen: How do we balance the healthcare system so that we help all patients, regardless of socioeconomic standing?}

Dr. Becker: Innovations on the supply side are going to play an important role. Improving public healthcare so that it is essentially either single payer or available to all will reduce expenditures. It is not a mystery; the world is full of countries that provide better basic healthcare to a larger population for less cost.

Dr. Padrón: Improving on the delivery of programs such as Medicaid would be a good start. Prior to the Affordable Care Act (ACA), Medicaid "take up" hovered around $50-60 \%$. This meant that about $40 \%$ of individuals that were eligible for Medicaid were not enrolled.

These individuals would also be likely not to have a usual source of care, would not perform recommended screenings, and ultimately may be in worse health, even when they could have accessed care. These numbers improved with the ACA, but paying attention to how we

$66 \overline{\text { Improving on }}$ the delivery of programs such as Medicaid would be a good start. 99 design and deliver healthcare access in a more efficient way would be a good start.

Dr. Becker: A good example of social class stratification in healthcare was found in a study looking at determinants of breast cancer survival in Kentucky using Surveillance, Epidemiology, and End Results (SEER) cancer data, in a relatively racially homogeneous population over many counties.

The surprise in findings was that the stage of diagnosis did not vary much by social class. However, survival decreased with lower social classes and those who were distant from major cancer treatment centers. This gets back to Norma's point that the ability to "take up" or to engage the system varies enormously by social class. Thus, supply-side interventions that make it easier to access the system lead to (a) significantly improved health outcomes, and (b) reduced costs because later-stage treatments are much more expensive than prevention and early-stage treatments.

\section{Dr. Chen: What are ways to encourage people to engage in the healthcare system?}

Dr. Padrón: Something that gets lost in the discussions about patient engagement is that the majority of individuals are relatively healthy over their life cycle. Simply speaking, individuals do not see themselves as patients. They see themselves as mothers, daughters, professionals, and so on, and not as "patients." 
One of the things that I advocate is engaging and meeting people where they are as individuals. I think mobile and digital technologies including wearables and digital decision support tools have a lot of potential for two-way engagement between healthcare and patients, especially if digital tools are used seamlessly, just the way we-as individuals-engage with technology every day for most aspects of our lives. For example, do you know what I check every day? I take the Southeastern Pennsylvania Transportation Authority into work, so I check when trains are coming every day or if there are any delays. Incorporating technological reminders and nudges may be part of the equation, but these are most effective if designed to be adapted to individual needs and preferences.

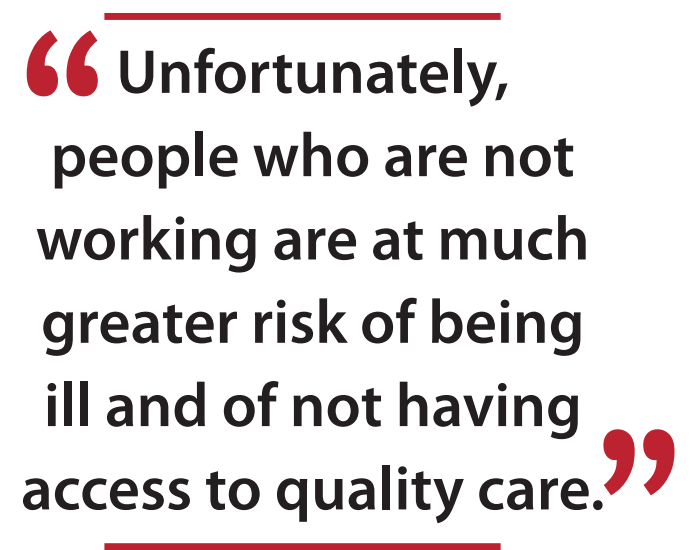

Dr. Becker: Healthcare would be easier to access if it were not workplace connected because people who get sick and gradually get ill may lose jobs. In 2030, I suspect that it still will be.

Unfortunately, people who are not working are at much greater risk of being ill and of not having access to quality care. A realistically ideal system would be one in which there are different levels of healthcare-where county public health departments provide initial screening and primary care, with those in need referred up as necessary and with supplemental private insurance to cover accelerated treatment or elective care.

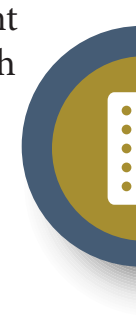

\section{Step 1: Reduce wasteful spending}

\section{Dr. Chen: How can we make the current healthcare system more efficient?}

Dr. Becker: Gradually, the role of nurses replacing doctors in many activities is an important cost-saving step, since you do not need MDs to do all things in medicine, including acting as initial interfaces. On the supply side, having more money allows the system to pour more resources into training nurses and other healthcare individuals, as opposed to producing more ultra-skilled, specialist MDs.

Dr. Padrón: In the context of the U.S. healthcare system, there is a lot of pressure to reduce wasteful spending. Roughly, spending on unnecessary, low value, and wasteful care accounts for about $30 \%$ of all healthcare spending. That would seem to indicate that there is a lot of room for improving efficiency in the healthcare system. The general perception is that there is a lack of coordination of services

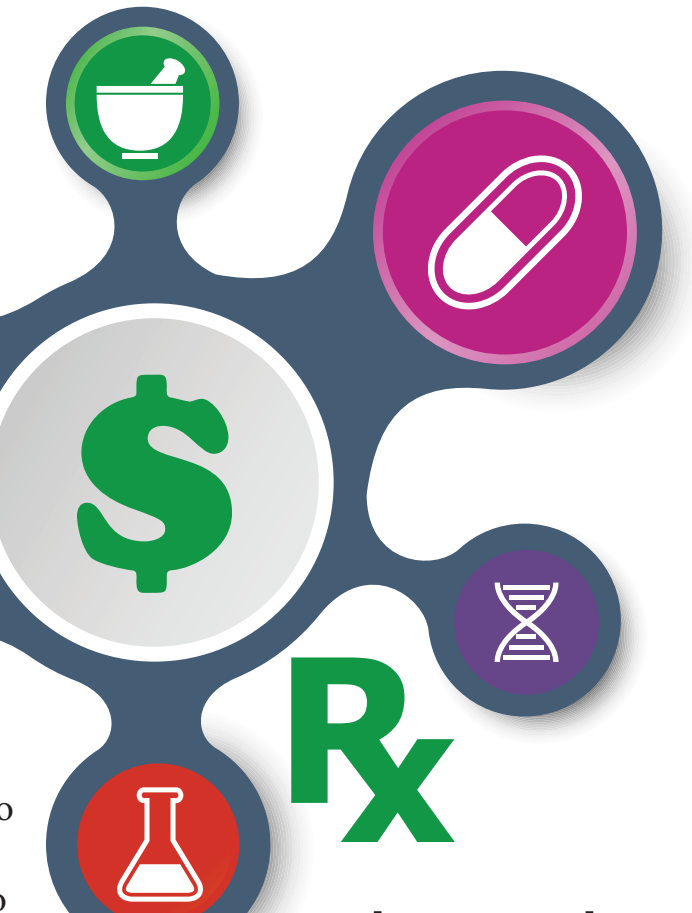

By the Numbers

More than $\mathbf{\$ 7 5 0}$ billion of U.S. healthcare spending annually represents waste, including approximately $\mathbf{\$ 2 0 0}$ billion in overtreatment. Source: JAMA Intern Med 2016;176:1567-1571 
in the healthcare system. However, the evidence on care coordination models is not consistent across the board in terms of generating a more efficient delivery of healthcare, at least in the form of cost savings.

One potentially low-cost and high-value way of achieving efficiencies in the current healthcare system could be to incorporate considerations of patients' living environment and circumstances systematically into delivery models of prevention and management. For instance, it is common to hear that better diet and exercise results in better health outcomes in the context of some chronic conditions. But how can a person engage in better diet and exercise if she lives in a place with no access to healthy foods, no transportation, and no open spaces?

This information should not be expensive or cumbersome to access for either patients or their healthcare providers, and can improve planning and treatment decisions. In a world where we care about health outcomes and not only about health processes, efficiency in the healthcare system means we need to move past knowing that zip codes determine how long and healthy a person lives to connecting the data points into actionable strategies.

Dr. Becker: This would require public health measures, such as adding social workers who would engage with and encourage people to lead healthier life-styles. Programs at the city or county level need to be implemented to encourage people simply to modify life-styles and seek preventive

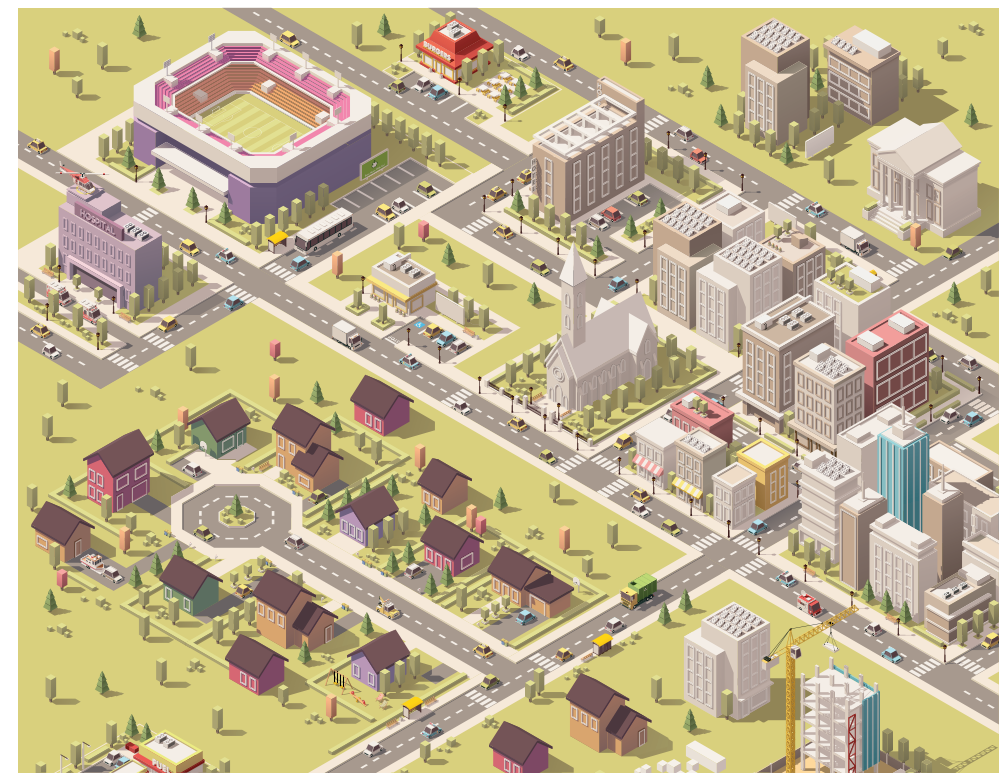
care, long before they go to clinics, see doctors, or show up at a hospital after a condition presents itself.

New programs will also utilize emerging technologies, as many young researchers are participating in health app startups to engage people in healthier activities.

Dr. Padrón: The public health aspect has the ability to meet citizens where they are. It is unfortunate that we just do not fund it enough.

\section{A message for President Trump}

\section{Dr. Chen: What would you tell the current administration to do to improve the present healthcare bill?}

Dr. Padrón: I would tell any administration to improve the data infrastructure and regulations that could make health data more accessible to patients that could
Social determinants of health are conditions in which people are born, grow, work, live, and age, and the wider set of forces and systems shaping the conditions of daily life. These forces and systems include economic policies and systems, development agendas, social norms, social policies, and political systems.

Source: The World Health Organization 
be shared in a secure way across systems (healthcare delivery, payers, etc.).

There have been huge investments into the digitization of health records and information technology from health systems, the federal government, and states. Today, $>90 \%$ of providers - individual practices and health systems - have digital health records. Unfortunately, there are enormous challenges of data interoperability across electronic health record vendors, which have hindered innovation. You would imagine that with this degree of digitization of health records, both personalized medicine and health should be much more advanced, but that is not the case.

Thus, today, we have a situation where it is still challenging for health systems, accountable care organizations, and payers to really understand the whole profile and evolution of people's health, and how health behaviors and outcomes associate with their social, economic, and living conditions. Everyone involved in the delivery of healthcare genuinely wants to understand the social determinants of health, but unfortunately the data environment is such that it is very difficult. Inevitably, the lack of data interoperability and the constraints on data sharing has slowed down innovation tremendously. In the context of digital health or mHealth, vendors of these solutions find it very difficult to integrate their solutions to most electronic medical record systems. This has meant that technologies that could in fact enable patient engagement are held at bay by the

66 Today, we have a situation where it is still challenging for health systems, accountable care organizations, and payers to really understand the whole profile and evolution of people's health. 99 current environment.

There are also concerns of compliance and the issues of data security that are still not solved. We have not yet found the right solutions for sharing data to and from patients, to and from health systems, and across sectors. As a health economist trying to design and evaluate population health strategies, I would like to underscore the urgency to figure out regulations and standards for multisectoral use, and sharing of data that will enable the innovations we need.

Dr. Becker: I would advise the current, or any, administration to explore global best practices. Saving overall costs will require a series of supply-side measures to get more trained staff per MD to reduce the capital intensity of initial contacts.

This is the opposite of going to the emergency department, which accounts for a significant-but not huge, as is sometimes asserted-proportion of additional expenses. I would also encourage competition to reduce costs. Having federally funded or federally supported rules that would encourage experimentation at state and local levels to determine a series of cost-saving practices and that could lead to improved outcomes would encourage innovation at the local level.

Dr. Padrón: The ACA actually did embed a lot of this local experimentation to establish evidence on best practices and payment models through the Centers 
of Medicare and Medicaid Services innovation center. Many of these models are ongoing.

For instance, Thomas JefFerson University is currently participating in the new oncology cancer model, which involves bundled payments or value-based models in the context of specialty care. This experimentation is difficult to deploy and implement, but early evidence shows very promising results, and it fits overarching goals to reduce costs and improve quality. I have not yet heard anyone say that they want higher costs and lower quality, right?

Since data and evidence are-in principle-nonpartisan, then experimentation models to establish evidence of what works should be a no-brainer. One of the arguments I have heard recently involved a discussion of Medicaid as an effective way to deliver healthcare. Most academic evidence actually points out that, indeed, Medicaid is hugely effective to deliver healthcare. But somehow, this is not known or understood. Again, I think that much of what the current administration can do to improve healthcare access is to enable evidence and information to be transparent.

Dr. Becker: I completely agree. By focusing on evidence-based improvements, gradual implementations, and public health/supply-side improvements, there will be changes that make America a much healthier place while maintaining or reducing costs. Ultimately, much of the improvements in health are going to come with interventions on health behaviors. Getting people to adopt healthy practices in their youth and early adulthood instead of waiting until it is essentially too late is also critical.

\section{- Big data for big fixes}

Dr. Chen: If you had access to unlimited patient data, what research study would you like to conduct?

Dr. Becker: I would like to study the long-run effects of less common conditions, but it all stems from getting access to data. When the Chernobyl disaster occurred, the USSR sent 400,000 people to build a sarcophagus over the stricken reactors. They kept track of how long people were exposed and, importantly, gave them supplemental pensions and improved healthcare access. The USSR's successor states have actually followed these people ever since 1986. They have to because they pay these so-called "liquidators" pensions and supplemental pensions, and improved healthcare was provided to those exposed, as well as their children and grandchildren. On the other hand, when radioactive plumes were released in the United States, our government went into denial instead of following up to see what happens to people who are exposed. While I have seen no systematic studies, the Russian health journals do contain papers that describe the health status of segments of those exposed. 
An example of an outstanding data set that is collected in the United States is the SEER data set mentioned earlier, which could be enormously improved if we had detailed individual information alongside it. My impression is that there are amazing databases in the United States, which is a key reason that more empirical health economics studies are focused on the United States than any other country, but that the data sets reflect administrative rather than research needs, again with some important exceptions.

Dr. Padrón: I would want to make sure that it contained data that enabled the systematic study of patients' living environments. A great start would be zip codes and environmental data.

Dr. Chirag Patel and his lab at Harvard are using openly available data sets on the exposome (http://biomedicalcomputationreview.org/content/takingexposome). I find the research on how the environment affects health very promising, and the current computational capacity and available open data will propel innovation in this area.

I think that establishing the evidence on whether and how incorporating information on the built environment and the effects of peer and social networks may improve health systems' ability to deliver care is important. The harder question perhaps is even if we had information on individuals' environment and relevant social and peer networks, would systems be able to deploy the resources? Beyond enabling the flow of information, what are the current challenges for delivering the last mile in personalized population health?

Dr. Becker: In addition to studying social networks and the environment, I would also add studying adherence. HII 